Pacific Journal of Mathematics

ON DETERMINATE HAMBURGER MOMENT PROBLEMS 


\section{ON INDETERMINATE HAMBURGER MOMENT PROBLEMS}

\section{T. S. ChiHARA}

This paper is concerned with the moment problems associated with a sequence of orthogonal polynominals defined by a recurrence formula. The principle interest centers on the question of the determinacy of the Stieltjes moment problem in the case where the corresponding Hamburger moment problem is indeterminate. Necessary and sufficient conditions expressed in terms of the recurrence formula are obtained for an indeterminate Hamburger moment problem to be a determined Stieltjes moment problem. Using this result, various criteria concerning the determinacy of the moment problems are obtained. It is also shown that if an indeterminate Hamburger moment problem has at least one solution whose spectrum is bounded below, then there is an extremal solution $\psi_{*}$ such that every substantially different solution has at least one spectral point smaller than the least spectral point of $\psi_{*}$.

Let $\left\{P_{n}(x)\right\}$ be a sequence of monic polynomials defined by a recurrence

$$
\begin{array}{rlr}
P_{n}(x) & =\left(x-c_{n}\right) P_{n-1}(x)-\lambda_{n} P_{n-2}(x), & n=1,2,3, \cdots \\
P_{-1}(x) & =0, P_{0}(x)=1 ; c_{n} \text { real, } \lambda_{n+1}>0 & (n \geqq 1) .
\end{array}
$$

Then it is a classical result that the $P_{n}(x)$ are orthogonal with respect to some mass distribution $d \psi(x)$ on the real line, $\psi$ being a bounded, nondecreasing function with an infinite spectrum.

In [2, Th. 1], it was shown that a necessary and sufficient condition that there is at least one such distribution function $\psi$ whose spectrum is a subset of $[0, \infty)$ is that $c_{n}>0(n \geqq 1)$ and that $\left\{\lambda_{n+1} / c_{n} c_{n+1}\right\}_{n=1}^{\infty}$ is a chain sequence. After an arbitrary choice of $\mu_{0}>0$, the orthogonality of the $P_{n}(x)$ determines a Hamburger moment sequence $\left\{\mu_{n}\right\}_{n=0}^{\infty}$. With the above chain sequence condition, this is also a Stieltjes moment sequence.

In [3], we gave some conditions on the coefficients in (1.1) by which the determinacy or indeterminacy of the Hamburger moment problem (HMP) can be decided. In the event the HMP is indeterminate, it is natural to inquire about the determinacy of the corresponding Stieltjes moment problem (SMP). In what follows, we will pursue this inquiry and apply our results and methods to a study of the uniqueness of the distributions obtained by Al-Salam and Carlitz [1] for a certain class of orthogonal " $q$-polynomials." 
2. Preliminaries. For the sake of definiteness (and without loss of generality), we will take $\mu_{0}=1$ for the moment sequence determined by (1.1). Thus we will speak of "the" moment problem associated with (1.1).

With the recurrence (1.1), we have the associated $J$-fraction

$$
\frac{\lambda_{1} \mid}{\mid x-c_{1}}-\frac{\lambda_{2} \mid}{\mid x-c_{2}}-\frac{\lambda_{3} \mid}{\mid x-c_{3}}-\cdots \quad\left(\lambda_{1}=\mu_{0}=1\right) .
$$

The $n$th approximant of $(2.1)$ is the rational function, $P_{n-1}^{(1)}(x) / P_{n}(x)$, where $\left\{P_{n}^{(1)}(x)\right\}$ is defined by (1.1) after replacing $c_{n}$ and $\lambda_{n}$ by $c_{n}^{(1)}=c_{n+1}$ and $\lambda_{n}^{(1)}=\lambda_{n+1}$, respectively.

If $x_{n 1}<x_{n 2}<\cdots<x_{n n}$ denote the zeros of $P_{n}(x)$, then it follows from the separation properties of the zeros of $P_{n}(x)$ and $P_{n+1}(x)$ that $\left\{x_{n i}\right\}_{n=i}^{\infty}$ is decreasing while $\left\{x_{n n}\right\}$ is increasing. We write

$$
\begin{aligned}
\xi_{i} & =\lim _{n \rightarrow \infty} x_{n i} \\
\xi_{\infty} & =\lim _{n \rightarrow \infty} x_{n n} .
\end{aligned} \quad i=1,2,3, \cdots
$$

Then $\left(\xi_{1}, \xi_{\infty}\right)$ is the so-called "true" interval of orthogonality of $\left\{P_{n}(x)\right\}$ and there is always one solution of the associated HMP whose spectrum is a subset of $\left[\xi_{1}, \xi_{\infty}\right]$. Also, $\xi_{1} \geqq c$ if and only if $c_{n}-c>0(n \geqq 1)$ and $\left\{\lambda_{n+1} /\left(c_{n}-c\right)\left(c_{n+1}-c\right)\right\}$ is a chain sequence [2, Lemma 5].

In case the HMP associated with (2.1) is also a solvable SMP, then (2.1) is the even part of the corresponding $S$-fraction (e.g. [5, p. 73])

$$
\frac{1 \mid}{\mid k_{1} z}+\frac{1 \mid}{\mid k_{2}}+\frac{1 \mid}{\mid k_{3} z}+\frac{1 \mid}{\mid k_{4}}+\cdots .
$$

The coefficients in the two continued fractions are related by the formulas (see $[9, \S 28]$ )

$$
\begin{aligned}
c_{n} & =-\left(b_{2 n-2}+b_{2 n-1}\right) \quad n=1,2,3, \cdots \\
\lambda_{n+1} & =b_{2 n-1} b_{2 n}
\end{aligned}
$$

where $b_{0}=0$ and $b_{i}=\left(k_{i} k_{i+1}\right)^{-1}(i \geqq 1)$ and $k_{1}=\lambda_{1}^{-1}=1$.

3. Stieltjes' condition. Stieltjes [6] (cf. also [5], Th. 2.20]) showed that a necessary and sufficient condition that the SMP associated with (2.3) (hence also with (2.1)) be determined is

$$
\sum_{i=1}^{\infty}\left|k_{i}\right|=\infty \text {. }
$$

From the relations (2.4) and (2.5), we obtain 


$$
\begin{aligned}
& k_{1}=1, \quad k_{2 n+1}=\frac{b_{1} b_{3} \cdots b_{2 n-1}}{b_{2} b_{4} \cdots b_{2 n}} \\
& k_{2}=\frac{1}{b_{1}}, \quad k_{2 n+2}=\frac{b_{2} b_{4} \cdots b_{2 n}}{b_{1} b_{3} \cdots b_{2 n+1}}, \quad n=1,2,3, \cdots .
\end{aligned}
$$

On the other hand, from relations in $[2, \S 2]$, we know that if $c_{n}>0$ and $\alpha=\left\{\lambda_{n+1} / c_{n} c_{n+1}\right\}$ is a chain sequence, then the associated moment problem is a solvable SMP and we have

$$
\begin{aligned}
c_{n} & =\gamma_{2 n-1}+\gamma_{2 n} \\
\lambda_{n+1} & =\gamma_{2 n} \gamma_{2 n+1}
\end{aligned} \quad \gamma_{1}=0, \gamma_{n}>0(n \geqq 2)
$$

where $\gamma_{2 k-1}=m_{k-1} c_{k}$ and $\left\{m_{k}\right\}_{0}^{\infty}$ is the minimal parameter sequence for $\alpha$. Comparing (2.4) and (3.3), we thus obtain

$$
\begin{gathered}
k_{1}=1, \quad k_{2 n+1}=\frac{c_{1}\left(1-m_{1}\right) \cdots\left(1-m_{n-1}\right)}{m_{1} m_{2} \cdots m_{n} c_{n+1}} \\
k_{2}=-\frac{1}{c_{1}}, \quad k_{2 n+2}=-\frac{m_{1} m_{2} \cdots m_{n}}{\left(1-m_{1}\right) \cdots\left(1-m_{n}\right)} .
\end{gathered}
$$

If we write $p_{n}(x)=\left(\lambda_{2} \cdots \lambda_{n+1}\right)^{-1 / 2} P_{n}(x)$, then $[3, \text { p. } 708]^{1}$

$$
\left[p_{n}(0)\right]^{2}=\frac{c_{1}\left(1-m_{1}\right) \cdots\left(1-m_{n-1}\right)}{m_{1} m_{2} \cdots m_{n} c_{n+1}} .
$$

Thus Stieltjes' (3.1) is equivalent to the divergence of at least one of the two series

$$
\sum_{n=1}^{\infty} \frac{m_{1} m_{2} \cdots m_{n}}{\left(1-m_{1}\right) \cdots\left(1-m_{n}\right)} \quad \text { and } \quad \sum_{n=1}^{\infty}\left[p_{n}(0)\right]^{2} .
$$

But by a theorem of Wall [9, Th. 19.3], a necessary and sufficient condition that $\alpha$ determine its parameters uniquely is that the first series in (3.4) diverge. Thus we can state the transformed criterion of Stieltjes as follows:

THEOREM 1. Let $c_{n}>0$ and let $\alpha=\left\{\lambda_{n+1} / c_{n} c_{n+1}\right\}$ be a chain sequence (so that the corresponding SMP has a solution). Then a necessary and sufficient condition that the SMP be determined is that either $\sum\left[p_{n}(0)\right]^{2}=\infty$ or $\alpha$ determines its parameters uniquely.

Throughout the remainder of this paper, we will maintain the notation, $\alpha=\left\{\lambda_{n+1} / c_{n} c_{n+1}\right\}$.

1 There is a factor of $c_{1}$ missing from the pertinent formula in the citation. This omission is carried through to a number of the following formulas but does not affect any of the conclusions drawn. 
Lemma 1. Let $c_{n}>0$ and let $\alpha$ be a chain sequence. Then

$$
\lim _{n \rightarrow \infty} \frac{P_{n+1}(0)}{P_{n}^{(1)}(0)}=-c_{1} M_{0}
$$

where $M_{0}$ denotes the "0th" maximal parameter for $\alpha$.

Proof. By a formula of Wall [9, (19.6)],

$$
M_{0}=1-\frac{\alpha_{1} \mid}{\mid 1}-\frac{\alpha_{2} \mid}{\mid 1}-\frac{\alpha_{3} \mid}{\mid 1}-\cdots, \quad \alpha_{n}=\frac{\lambda_{n+1}}{c_{n} c_{n+1}} .
$$

Comparing this with (2.1) (with $x=0$ ), we see that

$$
\left(-c_{1} M_{0}\right)^{-1}=\frac{\lambda_{1} \mid}{\mid-c_{1}}-\frac{\lambda_{2} \mid}{\mid-c_{2}}-\frac{\lambda_{3} \mid}{\mid-c_{3}}-\cdots=\lim _{n \rightarrow \infty} \frac{P_{n}^{(1)}(0)}{P_{n+1}(0)} .
$$

Lemma 1 is really needed in the next section but it is of some interest here in view of a theorem of Hamburger [5, Th. 2.17] that a necessary and sufficient condition for a HMP to be determined is the divergence of at least one of the series, $\sum\left[p_{n}(0)\right]^{2}$ and $\sum\left[p_{n}^{(1)}(0)\right]^{2}$, where $p_{n}^{(1)}(x)=\left(\lambda_{2}^{(1)} \cdots \lambda_{n+1}^{(1)}\right)^{-1 / 2} P_{n}^{(1)}(x)$ (cf. also [3, Th. 4.1]).

THEOREM 2. Let $c_{n}>0$ and let $\alpha$ be a chain sequence.

(A) If $\alpha$ does not determine its parameters uniquely, then the HMP and SMP are both determined or are both indeterminate according as $\sum\left[p_{n}(0)\right]^{2}$ diverges or converges.

(B) The case of a determined SMP which is an indeterminate HMP occurs if and only if $\alpha$ determines its parameters uniquely and $\sum\left[p_{n}^{(1)}(0)\right]^{2}<\infty$.

Proof. (A) If $\sum\left[p_{n}(0)\right]^{2}=\infty$, then by Hamburger's theorem, the HMP (and hence the SMP) is determined. Conversely, if the series converges and $\alpha$ does not determine its parameters uniquely, then by Theorem 1, the SMP (hence the HMP) is indeterminate.

(B) It follows that if the SMP is determined while the HMP is indeterminate, then $\alpha$ must determine its parameters uniquely. By Hamburger's theorem, we must also have $\sum\left[p_{n}^{(1)}(0)\right]^{2}<\infty$. Conversely, if the latter series converges, so does $\sum\left[p_{n}(0)\right]^{2}$ by Lemma 1 . Thus the HMP is indeterminate by Hamburger's theorem while the SMP will be determined according to Theorem 1 if $\alpha$ determines its parameters uniquely.

4. The case of an indeterminate HMP. We consider the polynomials $B_{n}(z)$ and $D_{n}(z)$ defined by 


$$
\begin{aligned}
& \lambda_{2} \cdots \lambda_{n+1} B_{n+1}(z)=P_{n+1}(z) P_{n-1}^{(1)}(0)-P_{n}(z) P_{n}^{(1)}(0) \\
& \lambda_{2} \cdots \lambda_{n+1} D_{n+1}(z)=P_{n+1}(z) P_{n}(0)-P_{n}(z) P_{n+1}(0) .
\end{aligned}
$$

Using the fact that $B_{n}(0)=-1[5,(2.46)]$, we obtain

$$
P_{n}(z)=P_{n-1}^{(1)}(0) D_{n+1}(z)-P_{n}(0) B_{n+1}(z) .
$$

(Note that we write $\lambda_{k}, P_{n}(z)$ and $P_{n}^{(1)}(z)$ for $\beta_{k-1}, Q_{n}(z)$ and $P_{n+1}(z)$, respectively, in [5].)

If we now assume that the HMP associated with (1.1) is indeterminate, then $B_{n}(z)$ and $D_{n}(z)$ converge uniformly on bounded subsets of the complex plane to entire functions $B(z)$ and $D(z)$ [5, Th. 2.11]. Hence if we now also assume that $c_{n}>0$ and $\alpha$ is a chain sequence, then by Lemma 1.

$$
\lim _{n \rightarrow \infty} \frac{P_{n}(z)}{P_{n-1}^{(1)}(0)}=D(z)+c_{1} M_{0} B(z)
$$

uniformly on bounded subsets of the complex plane.

Now by a theorem of Nevanlinna [5, Th. 2.13], the zeros of the entire function $E(z)=D(z)+c_{1} M_{0} B(z)$ are real, simple and coincide with the spectrum of one of the extremal solutions, $\psi_{*}$, of the HMP.

Since the convergence in (4.1) is uniform, it follows from (2.2) that each $\xi_{i}$ is a zero of $E(z)$ and hence also that $\xi_{i}<\xi_{i+1}\left(\xi_{1} \geqq 0\right)$. It then follows from Hurwitz' theorem on uniform limits of analytic functions [8, Th. 1.91.3] that $\left\{\xi_{i} \mid i=1,2,3, \cdots\right\}$ is the spectrum of the extremal solution, $\psi_{*}$.

We summarize with a lemma which will be sharpened somewhat subsequently (Theorem 5).

LEMMA 2. Let $c_{n}>0$ and let $\alpha$ be a chain sequence. If the associated HMP is indeterminate, then there is an extremal solution $\psi_{*}$ whose spectrum coincides with the set $\left\{\xi_{i} \mid i=1,2,3, \cdots\right\}$. The spectrum of every solution of the HMP which is not substantially equal to $\psi_{*}$ contains at least one point that does not exceed $\xi_{1}$.

Proof. Only the last assertion requires further comment. (By "substantially equal" solutions of the HMP, we mean, following Shohat and Tamarkin [5], solutions with the same points of continuity whose difference is constant at all points of continuity.) Since the zeros of $P_{n}(x)$ must lie in the interior of the interval of orthogonality, then $\xi_{1}$ must belong to the smallest closed interval containing the support of any $d \psi(x)$ with respect to which the $P_{n}(x)$ are orthogonal.

Theorem 3. If the true interval of orthogonality for (1.1) is 
$(0, \infty)$ and if the associated HMP is indeterminate, then the chain sequence $\alpha$ determines its parameters uniquely.

Proof. Assume $\alpha$ does not determine its parameters uniquely. Then [2, Th. 1] the $P_{n}(x)$ are "kernel polynomials" - that is, there is a distribution function $\varphi$ whose spectrum is a subset of $[0, \infty)$ such that the $P_{n}(x)$ are orthogonal with respect to $x d \varphi(x)$. Further, by Theorem 2, the corresponding SMP is also indeterminate.

By [2, Th. 2], there is a continuum of indeterminate SMPs whose solutions have the property described for $\varphi$ above. Consider one of these SMPs. By Lemma 2, this indeterminate SMP-which is also an indeterminate HMP - has an extremal solution $\varphi_{*}$ whose spectrum is a well ordered subset of $[0, \infty)$.

It then follows that the spectrum of the distribution function $\psi_{*}$ defined by $d \psi_{*}(x)=x d \varphi_{*}(x)$ does not contain 0 , hence must contain a least element $a>0$. But the $P_{n}(x)$ are orthogonal with respect to $d \psi_{*}(x)$ which means the true interval of orthogonality is $(a, \infty)$, contrary to hypothesis.

THEOREM 4. If the HMP associated with (1.1) is indeterminate, then the corresponding SMP is (solvable and) determined if and only if the true interval of orthogonality is $(0, \infty)$.

Proof. If the SMP is solvable and determined, then $\alpha$ is a chain sequence which by Theorem 2 determines its parameters uniquely. According to [2, Th. 1], the $P_{n}(x)$ are not "kernel polynomials" so the true interval of orthogonality must be $(0, \infty)$.

Conversely, suppose the true interval of orthogonality is $(0, \infty)$. Since the HMP is indeterminate, it follows from Theorem 3 that $\alpha$ must determine its parameters uniquely. Thus by Theorem 1, the SMP is determined.

We can now restate Lemma 2 in stronger form.

TheOREM 5. Assume there is a real c such that $c_{n}-c>0$ and $\left\{\lambda_{n+1} /\left(c_{n}-c\right)\left(c_{n+1}-c\right)\right\}$ is a chain sequence. Suppose also that the associated HMP is indeterminate. Then the HMP has an extremal solution $\psi_{*}$ whose spectrum is the set $\left\{\xi_{i} \mid i=1,2,3, \cdots\right\}$. The spectrum of every solution not substantially equal to $\psi_{*}$ contains at least one point smaller than $\xi_{1}$.

Proof. By hypothesis, $\xi_{1}$ is finite $\left(\xi_{1} \geqq c\right)$. If we put $\pi_{n}(x)=$ $P_{n}\left(x+\xi_{1}\right)$, the $\pi_{n}(x)$ are orthogonal with respect to $d \psi\left(x+\xi_{1}\right)$, where $\dot{\psi}$ is any solution of the original moment problem, and the corresponding true interval of orthogonality is $(0, \infty)$. 
If the original HMP is indeterminate, so is the HMP associated with the $\pi_{n}(x)$. By Lemma 2, the latter HMP has an extremal solution $\varphi_{*}$ whose spectrum coincides with the set $\left\{\xi_{i}-\xi_{1} \mid i=1,2, \cdots\right\}$ determined by the zeros of $\left\{\pi_{n}(x)\right\}$.

But since the true interval of orthogonality is $(0, \infty)$, then by Theorem 4, the corresponding SMP is determined. Then $\varphi_{*}$ is substantially the only solution of the transformed HMP whose spectrum is a subset of $[0, \infty)$. Thus in terms of the original HMP, $\psi_{*}(x)=$ $\varphi_{*}\left(x-\xi_{1}\right)$ has the desired properties.

COROLLARY. If an indeterminate HMP is a determined SMP, then the solution of the SMP is an extremal solution of the HMP whose spectrum contains 0 and is a discrete, unbounded subset of $[0, \infty)$.

Proof. By Theorem 4, the true interval of orthogonality is $\left(\xi_{1}, \xi_{\infty}\right)=(0, \infty)$.

The preceding shows that-at least from the viewpoint of orthogonal polynomials-when the HMP is indeterminate, the question of the determinacy of the SMP is relatively unimportant since determinacy depends only on an "accident" of translation (whereas determinacy of the HMP is invariant under translation). Stieltjes had observed that in the indeterminate case, $\xi_{1}>0[6$, p. 441]. However, it does not seem to have been observed previously that there is always substantially only one solution of a SMP whose spectrum is a subset of $\left[\xi_{1}, \infty\right)$.

In a sense, the solution described in Theorem 5 is a "natural" one since every spectral point is a limit point of a sequence of zeros of the $P_{n}(x)$, a property that is shared by the solution of a determined HMP (Stone [7, Th. 10.42]).

In [2, Th. 8] and [3, Th. 3.1], we have obtained sufficient conditions (in terms of $c_{n}$ and $\lambda_{n}$ ) for the spectrum associated with (1.1) to be a discrete, unbounded set. These conditions are accompanied by the hypothesis that the associated HMP is determined but in view of the above, this hypothesis can be dropped if we think in terms of the "natural" solution. With this viewpoint, a recent theorem of Maki on continued fractions [4, Th. 3.2] should be compared with [2, Th. 8].

5. Examples. We illustrate various aspects of the preceding theory with two examples. have

(A) The Laguerre polynomials. Using the notation of [8], we 


$$
\begin{array}{cc}
P_{n}(x)=(-1)^{n} L_{n}^{(\alpha)}(x) & (\alpha>-1) \\
c_{n}=2 n+\alpha-1, \quad \lambda_{n+1}=n(n+\alpha) & (n \geqq 1) .
\end{array}
$$

Then

$$
\begin{aligned}
\alpha_{n} & =\frac{\lambda_{n+1}}{c_{n} c_{n+1}}=\frac{n(n+\alpha)}{(2 n+\alpha-1)(2 n+\alpha+1)} \\
& =\left(1-\frac{n-1}{2 n+\alpha-1}\right) \frac{n}{2 n+\alpha+1} .
\end{aligned}
$$

It follows from Wall's criterion (following (3.4)) that $\left\{\alpha_{n}\right\}$ is a chain sequence which determines its parameters, $m_{k}=k /(2 n+\alpha-1)(k \geqq 0)$, uniquely if and only if $\alpha \leqq 0$.

Then for $-1<\alpha \leqq 0$, the moment problem is at least a determined SMP (by Theorem 1) and the true interval of orthogonality is $(0, \infty)$. Since the weight function for the Laguerre polynomials is $x^{\alpha} e^{-x}$ and this does not correspond to an extremal solution, it follows from Theorem 5, corollary, that the HMP must be determined for $-1<\alpha \leqq 0$. Then, also, the corresponding spectrum is $[0, \infty)$ and the zeros of the polynomials are dense in $(0, \infty)$.

Now the $L_{n}^{(\alpha+1)}(x)$ are the kernel polynomials corresponding to the $L_{n}^{(\alpha)}(x)$ so it follows that the true interval of orthogonality must be $(0, \infty)$ for $0<\alpha \leqq 1$ and hence for all $\alpha>-1$ by induction. Since for $\alpha>0,\left\{\alpha_{n}\right\}$ does not determine its parameters uniquely, Theorem 3 shows that the HMP must be determined for $\alpha>0$ also.

Thus the HMP is determined for every $\alpha>-1$ and by Riesz' theorem [5, Th. 2.14], the Laguerre polynomials are complete in the corresponding $L^{2}$ space (as is well known).

(B) Al-Salam and Carlitz [1] have recently considered two interesting classes of orthogonal polynomials involving " $q$-numbers." One of these satisfies the recurrence

$$
\begin{gathered}
V_{n+1}^{(a)}(x)=\left[x-(1+a) q^{-n}\right] V_{n}^{(a)}(x)-a q^{1-2 n}\left(1-q^{n}\right) V_{n-1}^{(a)}(x) \quad(n \geqq 0) \\
V_{-1}^{(a)}(x)=0, V_{0}^{(a)}(x)=1 ; \quad 0<q<1, a>0 .
\end{gathered}
$$

These authors obtain the corresponding moments and construct a solution of the moment problem. (However, their solution is not non-decreasing when $a q>1$ and does not exist if $a q^{k}=1$.) The other system considered by them has bounded coefficients in its recurrence and hence the corresponding HMP is automatically determined. For the $V_{n}^{(a)}(x)$, however, the HMP may be indeterminate. Since they did not investigate the uniqueness of the solution of the moment problem, such a study will provide an interesting application of our methods.

Relative to (1.1), we have 


$$
\begin{aligned}
c_{n} & =(1+a) q^{1-n}, \quad \lambda_{n+1}=a q^{1-2 n}\left(1-q^{n}\right) \\
\alpha_{n} & =\lambda_{n+1} /\left(c_{n} c_{n+1}\right)=a(1+a)^{-2}\left(1-q^{n}\right) .
\end{aligned}
$$

Since $\alpha_{n}<1 / 4,\left\{\alpha_{n}\right\}$ is a chain sequence [9, Th, 20.1] which does not determine its parameters uniquely [9, Th. 19.6].

Next let $v_{n}^{(a)}(x)=\left(\lambda_{2} \cdots \lambda_{n+1}\right)^{-1 / 2} V_{n}^{(a)}(x)$. With the aid of [1, (4.8)], we find

$$
\left[v_{n}^{(\alpha)}(0)\right]^{2}=a^{-n} q^{n}(q)_{n}^{-1}\left[H_{n}(\alpha)\right]^{2}, \quad(q)_{n}=(1-q) \cdots\left(1-q^{n}\right),
$$

where

$$
\sum_{r=0}^{n} a^{r}<H_{n}(a) \equiv \sum_{r=0}^{n} \frac{(q)_{n}}{(q)_{n-r}(q)_{r}} a^{r}<(q)_{n}^{-1} \sum_{r=0}^{n} a^{r} .
$$

It follows that $\sum\left[v_{n}^{(a)}(0)\right]^{2}<\infty$ if and only if $0<q<a \leqq 1$ or $1<a<q^{-1}$. Since $\alpha=\left\{\alpha_{n}\right\}$ does not determine its parameters uniquely, Theorem 2 shows that:

(a) both the SMP and HMP are determined if

$$
0<a \leqq q<1 \text { or } 1<q^{-1} \leqq a ;
$$

(b) both the SMP and HMP are indeterminate if

$$
0<q<a \leqq 1 \text { or } 1<a<q^{-1} \text {. }
$$

Except for the cases, $a=q$ and $a=q^{-1}$, the above results can also be obtained using [3, Th. 4.3].

In the indeterminate case, there may some interest in knowing whether the distribution function obtained by Al-Salam and Carlitz is the extremal solution described in Theorem 5 .

To this end, translate the minimal spectral point, 1 , of the distribution function, $\beta=\beta^{a}$, obtained in [1] to the origin. That is, consider (1.1) with $c_{n}$ replaced by $c_{n}^{\prime}=c_{n}-1=(1+a) q^{1-n}-1$. Then

$$
\alpha_{n}^{\prime}=\frac{\lambda_{n+1}}{c_{n}^{\prime} c_{n+1}^{\prime}}=\frac{a\left(1-q^{n}\right)}{\left(1+a-q^{n-1}\right)\left(1+a-q^{n}\right)}
$$

and it is readily verified that $\left\{\alpha_{n}^{\prime}\right\}$ is a chain sequence with minimal parameters $m_{k}=\left(1-q^{k}\right)\left(1+a-q^{k}\right)^{-1}(k \geqq 0)$. Wall's criterion shows that $\left\{\alpha_{n}^{\prime}\right\}$ determines its parameters uniquely if and only if $0<a \leqq 1$. By theorem 2, the translated SMP is determined (and the HMP is indeterminate) if and only if $0<a \leqq 1$. In terms of the original moment problem, this means that the true interval of orthogonality is $(1, \infty)$.

Summarizing:

(i) if $0<a \leqq q<1$ or $1<q^{-1} \leqq a$, the solution of the HMP is substantially unique (and is given by $\beta$ in [1] when $0<a \leqq q<1$ ); 
(ii) if $0<q<a \leqq 1$, the solution $\beta$ is not substantially unique but it is substantially the only solution whose spectrum is a subset of $[1, \infty)$;

(iii) If $1<a<q^{-1}$, there are infinitely many solutions of the moment problem whose minimal spectral point is larger than 1 (that is, the true interval of orthogonality is $(c, \infty)$ where $c>1)$.

\section{REFERENCES}

1. W. A. Al-Salam and L. Carlitz, Some orthogonal q-polynomials, Math. Nachr. 30 (1965), 47-61.

2. T. S. Chihara, Chain sequences and orthogonal polynomials, Trans. Amer. Math. Soc. 104 (1962), 1-16.

3. - On recursively defined orthogonal polynomials, Proc. Amer. Math. Soc. 16 (1965), 702-710.

4. D. P. Maki, On constructing distribution functions; with applications to Lommel polynomials and Bessel functions, Trans. Amer. Math. Soc. 130 (1968), 281-297.

5. J. Shohat and J. Tamarkin, The Problem of Moments, Math. Surveys Mo. 1, Amer. Math. Soc., New York, 1943, 1950.

6. T. J. Stieltjes, Recherches sur les fractions continues, Oevres, Tome II, Noordhoff, Groningen, 1918.

7. M. H. Stone, Linear Transformations in Hilbert Space, Amer. Math. Soc. Colloq. Publ., vol. 15, Amer. Math. Soc., New York, 1932.

8. G. Szegö, Orthogonal Polynomials, Amer. Math. Soc. Colloq. Publ., vol. 23, Amer. Math. Soc., New York, 1939.

9. H. S. Wall, Analytic Theory of Continued Fractions, van Nostrand, New York, 1948.

Received January 9, 1967. This research was supported by the National Science Foundation (GP 4347).

Seattle University 


\section{PACIFIC JOURNAL OF MATHEMATICS}

\section{EDITORS}

\section{H. ROYDEN}

Stanford University

Stanford, California

\section{J. Dugundu}

Department of Mathematics University of Southern California Los Angeles, California 90007

RICHARD ARENS

University of California Los Angeles, California 90024

ASSOCIATE EDITORS
E. F. Beckenbach
B. H. NEUMANN
F. WoLF
K. YosidA

\section{SUPPORTING INSTITUTIONS}

\author{
UNIVERSITY OF BRITISH COLUMBIA \\ CALIFORNIA INSTITUTE OF TECHNOLOGY \\ UNIVERSITY OF CALIFORNIA \\ MONTANA STATE UNIVERSITY \\ UNIVERSITY OF NEVADA \\ NEW MEXICO STATE UNIVERSITY \\ OREGON STATE UNIVERSITY \\ UNIVERSITY OF OREGON \\ OSAKA UNIVERSITY \\ UNIVERSITY OF SOUTHERN CALIFORNIA
}

STANFORD UNIVERSITY

UNIVERSITY OF TOKYO

UNIVERSITY OF UTAH

WASHINGTON STATE UNIVERSITY

UNIVERSITY OF WASHINGTON

AMERICAN MATHEMATICAL SOCIETY
CHEVRON RESEARCH CORPORATION
TRW SYSTEMS

AMERICAN MATHEMATICAL SOCIETY

TRW SYSTEMS

NAVAL WEAPONS CENTER

Mathematical papers intended for publication in the Pacific Journal of Mathematics should be in typed form or offset-reproduced, double spaced with large margins. Underline Greek letters in red, German in green, and script in blue. The first paragraph or two must be capable of being used separately as a synopsis of the entire paper. It should not contain references to the bibliography. Manuscripts, in duplicate if possible, may be sent to any one of the four editors. All other communications to the editors should be addressed to the managing editor, Richard Arens, University of California, Los Angeles, California 90024.

Each author of each article receives 50 reprints free of charge; additional copies may be obtained at cost in multiples of 50 .

The Pacific Journal of Mathematics is published monthly. Effective with Volume 16 the price per volume (3 numbers) is $\$ 8.00$; single issues, $\$ 3.00$. Special price for current issues to individual faculty members of supporting institutions and to individual members of the American Mathematical Society: $\$ 4.00$ per volume; single issues $\$ 1.50$. Back numbers are available.

Subscriptions, orders for back numbers, and changes of address should be sent to Pacific Journal of Mathematics, 103 Highland Boulevard, Berkeley 8, California.

Printed at Kokusai Bunken Insatsusha (International Academic Printing Co., Ltd.), 7-17, Fujimi 2-chome, Chiyoda-ku, Tokyo, Japan.

PUBLISHED BY PACIFIC JOURNAL OF MATHEMATICS, A NON-PROFIT CORPORATION

The Supporting Institutions listed above contribute to the cost of publication of this Journal, but they are not owners of publishers and have no responsibility for its content or policies. 


\section{Pacific Journal of Mathematics \\ Vol. 27, No. 3}

March, 1968

Charles A. Akemann, Invariant subspaces of $C(G) \ldots \ldots \ldots \ldots \ldots \ldots . \ldots 41$

Dan Amir and Zvi Ziegler, Generalized convexity cones and their duals ... . 425

Raymond Balbes, On ( $J, M, \mathrm{~m})$-extensions of order sums of distributive



Jan-Erik Björk, Extensions of the maximal ideal space of a function algebra ........................................ 453

Frank Castagna, Sums of automorphisms of a primary abelian group ...... 463

Theodore Seio Chihara, On determinate Hamburger moment problems ..... .

Zeev Ditzian, Convolution transforms whose inversion function has complex

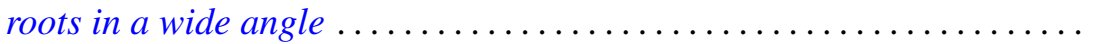

Myron Goldstein, On a paper of Rao .

Velmer B. Headley and Charles Andrew Swanson, Oscillation criteria for

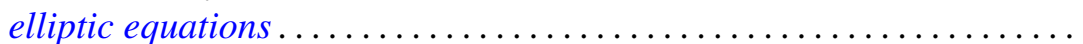

John Willard Heidel, Qualitative behavior of solutions of a third order nonlinear differential equation............................

Alan Carleton Hindmarsh, Pick's conditions and analyticity.............

Bruce Ansgar Jensen and Donald Wright Miller, Commutative semigroups

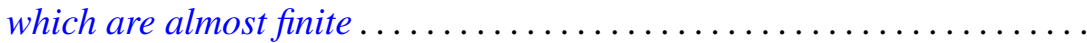

Lynn Clifford Kurtz and Don Harrell Tucker, An extended form of the

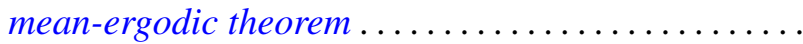

S. P. Lloyd, Feller boundary induced by a transition operator ...

Henry B. Mann, Josephine Mitchell and Lowell Schoenfeld, A new proof of the maximum principle for doubly-harmonic functions ...

Robert Einsohn Mosher, The product formula for the third obstruction ..

Sam Bernard Nadler, Jr., Sequences of contractions and fixed points ...

Eric Albert Nordgren, Invariant subspaces of a direct sum of weighted shifts...

Fred Richman, Thin abelian p-groups ...

Jordan Tobias Rosenbaum, Simultaneous interpolation in $\mathrm{H}_{2}$. II ...

Charles Thomas Scarborough, Minimal Urysohn spaces .

Malcolm Jay Sherman, Disjoint invariant subspaces..... .

Joel John Westman, Harmonic analysis on groupoids....

621

William Jennings Wickless, Quasi-isomorphism and TFM

Minoru Hasegawa, Correction to "On the convergence of resolvents of operators" 